\title{
Cinemómetro piezoeléctrico de alta exactitud (VUAE)
}

\author{
F. J. JIMÉNEZ MARTíNEZ', J. DE FRUTOS², C. PASTOR², M. VÁZQUEZ RODRíGUEZ ${ }^{1}$ \\ 'Dep. Sistemas Electrónicos y de Control, EUITT-UPM. Ctra. Valencia Km 7. 28031 Madrid, España. \\ 1,2 POEMMA-CEMDATIC. ETSIT-UPM. Ciudad Universitaria s/n. 28040 Madrid, España.
}

\begin{abstract}
Se ha desarrollado un sistema electrónico computerizado, portátil y de bajo consumo, denominado Medidor de Velocidad de Vehículos por Ultrasonidos de Alta Exactitud, VUAE. La alta exactitud de la medida conseguida en el VUAE hace que pueda servir de medida de referencia de la velocidad de un vehículo circulando en carretera. Por tanto el VUAE puede usarse como medida de referencia que permita estimar el error de los cinemómetros comerciales. El VUAE está compuesto por $\mathrm{n}(\mathrm{n} \geq 2)$ parejas de emisores y receptores piezoeléctricos de ultrasonidos, denominados E- $\mathrm{R}_{\mathrm{ult}}$. Los emisores de las $\mathrm{n}$ parejas E- $\mathrm{R}_{\mathrm{ult}}$ generan n barreras de ultrasonidos, y los receptores piezoeléctricos captan la señal de los ecos cuando el vehículo atraviesa las barreras. Estos ecos se procesan digitalmente para conseguir señales representativas. Posteriormente, utilizando la técnica de la correlación cruzada de señales, se ha podido estimar con alta exactitud la diferencia de tiempos entre los ecos captados en cada barrera. Con los tiempos entre ecos y con la distancia entre cada una de las n barreras de ultrasonidos se puede realizar una estimación de la velocidad del vehículo con alta exactitud. El VUAE se ha contrastado con un sistema de velocidad de referencia, basado en cables piezoeléctricos.
\end{abstract}

Palabras clave: Material Piezoeléctrico, Aplicaciones con piezoeléctricos, Sistemas de medida, Cinemómetro

\section{High Accuracy Piezoelectric Kinemometer}

We have developed a portable computerized and low consumption, our system is called High Accuracy Piezoelectric Kinemometer measurement, herein VUAE. By the high accuracy obtained by VUAE it make able to use the VUAE to obtain references measurements of system for measuring Speeds in Vehicles. Therefore VUAE could be used how reference equipment to estimate the error of installed kinemometers. The VUAE was created with $\mathrm{n}(n \geq 2)$ pairs of ultrasonic transmitter-receiver, herein $E-R_{u l t}$. The transmitters used in the $n$ couples $E-R_{\text {lt }}$ generate $n$ ultrasonic barriers and receivers receive the echoes when the vehicle crosses the barriers. Digital processing of the echoes signals let us to obtain acceptable signals. Later, by mean of cross correlation technics is possible make a highly exact estimation of speed of the vehicle. The log of the moments of interception and the distance between each of the $\mathrm{n}$ ultrasounds allows for a highly exact estimation of speed of the vehicle. VUAE speed measurements were compared to a speed reference system based on piezoelectric cables.

Keywords: Piezoelectric material, Piezoelectric Applications, Measurement Systems, Kinemometer

\section{INTRODUCCIÓN}

Los sistemas de medida de velocidad de referencia habitualmente están ubicados en instalaciones fijas en los laboratorios de metrología, por lo que para realizar la calibración o estimación del error de un cinemómetro comercial de control de velocidad que está operativo en campo, implica desinstalarlo, transportarlo al laboratorio de metrología, calibrarlo y volverlo a instalar en la ubicación de origen. Existen sistemas de medida de velocidad de referencia móviles pero requieren una instalación engorrosa.

En el sistema que se presenta en este artículo, el sistema es portátil, con un consumo reducido y de instalación fácil y rápida en la zona de influencia de un cinemómetro comercial instalado en cualquier carretera.

\section{ESTADO DE LA TÉCNICA}

Actualmente las metodologías utilizadas para la medida de referencia de la velocidad de un vehículo se basan en la tecnología láser y en el mismo principio físico: medición del tiempo que tarda un móvil para recorrer una distancia conocida. Existen dos técnicas ampliamente utilizadas.

La filosofía de la primera de las técnicas consiste en la intercepción de dos barreras láser (1 y 2) por el vehículo en movimiento. Las barreras láser están separadas entre sí una distancia conocida $d_{b^{\prime}}$ y se registran los instantes de intercepción de ambas barreras $t_{1}$ y $t_{2}$. Dichas barreras se forman instalando un emisor de luz láser a un lado de la vía y un receptor o sensor láser al otro lado de la vía. Si se mide el tiempo entre intercepciones $t_{2}-t_{1}$ con la suficiente resolución se puede obtener una medida de la velocidad con suficiente exactitud como para ser usada como medida de referencia según la Ec. 1. 


$$
v=\frac{d_{b}}{t_{2}-t_{1}}
$$

La filosofía de la segunda técnica [1] se describe en la FIG. 1. Consiste en colocar $n$ sensores láser $(n \geq 2)$ en el arcén distanciados una distancia $d_{i+1, i}(i=1,2, \ldots, n-1)$ conocida y un emisor láser instalado en un vehículo de prueba. Cuando el haz de luz láser instalado sobre el vehículo de prueba incide sobre los sensores, se registran los instantes de tiempo de dichas incidencias $t_{i}$ pudiendo calcular el tiempo entre ellas, $t_{i+1}-t_{i}$. Se calcula la velocidad mediante la Ec. 2.

$$
v=\frac{\sum_{i=1}^{n-1} \frac{d_{i+1, i}}{t_{i+1}-t_{i}}}{n}
$$

Ambas metodologías presentan algunos problemas prácticos:

- Es necesaria una alineación previa entre el emisor y el receptor láser para poder interceptar el paso del vehículo. Esto implica un gasto excesivo de tiempo en el ajuste de la alineación antes de comenzar el proceso de medida.

- El pico de consumo de corriente de los sistemas láser es elevado, por lo que en el caso de uso de baterías se limita el tiempo de operación del sistema.

En el caso de la primera metodología hay que colocar equipamiento en ambos lados de la vía. Esto implica algunos aspectos negativos:

Existe un índice de peligrosidad añadido porque el/ los operario/s tienen que cruzar la vía para colocar el equipamiento.

La alimentación eléctrica al equipamiento en el arcén interior (arcén izquierdo en los países en los que se circula por la derecha), ha de llevarse con cable desde el otro arcén o colocando una batería autónoma.

La fijación entre el emisor y el receptor a su soporte y este al suelo debe ser lo suficientemente fuerte para que vibraciones externas al sistema no desalineen el emisor y el receptor láser. Tales vibraciones pueden ser provocadas por un vehículo pesado.

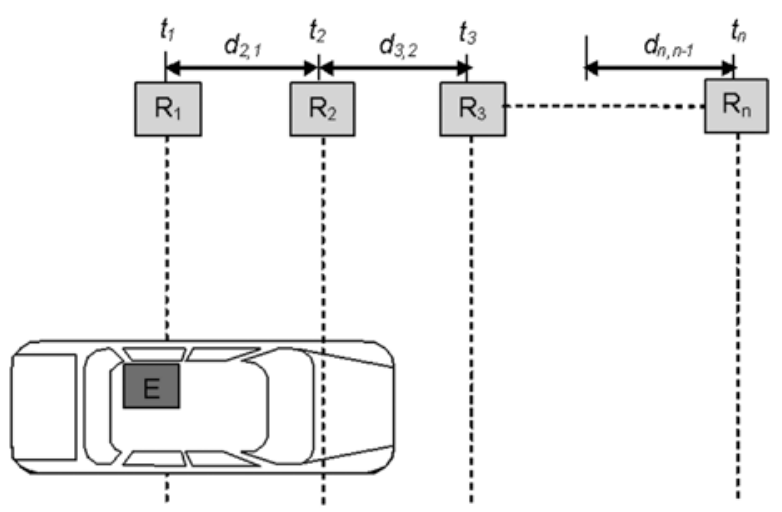

FIG. 1: Sistema basado en vehículo de prueba
En el caso de la segunda metodología:

Sólo se pueden medir velocidades si pasa por la vía el vehículo de referencia con un emisor láser instalado y alineado con los receptores láser.

La fijación del receptor a su soporte y este al suelo debe ser lo suficientemente fuerte para que vibraciones externas al sistema no desalineen el emisor y el receptor láser. Tales vibraciones pueden ser provocadas por un vehículo pesado.

\section{REQUERIMIENTOS DEL SISTEMA}

Después de esta visión global del estado del arte, nuestra motivación es la de construir un sistema que no esté basado en tecnología láser. El sistema propuesto estará basado en el uso de piezoeléctricos [2] emitiendo una señal de ultrasonidos y captando los ecos recibidos y debe cubrir las debilidades expuestas en el punto anterior. Para ello se propone que el sistema cumpla los siguientes requerimientos:

- El VUAE debe ser capaz de medir la velocidad de un vehículo en movimiento con una exactitud mejor que $0,5 \mathrm{~km} / \mathrm{h}$ en un rango de velocidades de 0 a 300 $\mathrm{km} / \mathrm{h}$.

- $\quad$ El VUAE debe ser capaz generar $n(n \geq 2)$ barreras de ultrasonidos, generadas por emisores de ultrasonidos y recibir el eco en un transductor de ultrasonidos al paso del vehículo en movimiento. La detección debe ser posible cuando el vehículo pase a 5 metros o menos de la barrera.

- El VUAE medirá la medida de la velocidad inmediatamente después de que el vehículo sea detectado en al menos 2 barreras de las $n(n \geq 2)$ barreras de ultrasonidos.

- En el VUAE las barreras deben estar paralelas entre sí con un error de $\pm 1^{\circ}$.

- La n barreras del VUAE serán de instalación portátil, aunque no es excluyente el hecho de que puedan instalarse en un laboratorio permanente. Dichas barreras deben poder colocarse alineadas y de forma rápida y sencilla.

- El consumo eléctrico del VUAE debe ser inferior a $200 \mathrm{~mA}$.

El sistema que se describe en este artículo se ha diseñado en base a los requerimientos anteriores, en la FIG. 2 se muestra un esquema de bloques del VUAE.

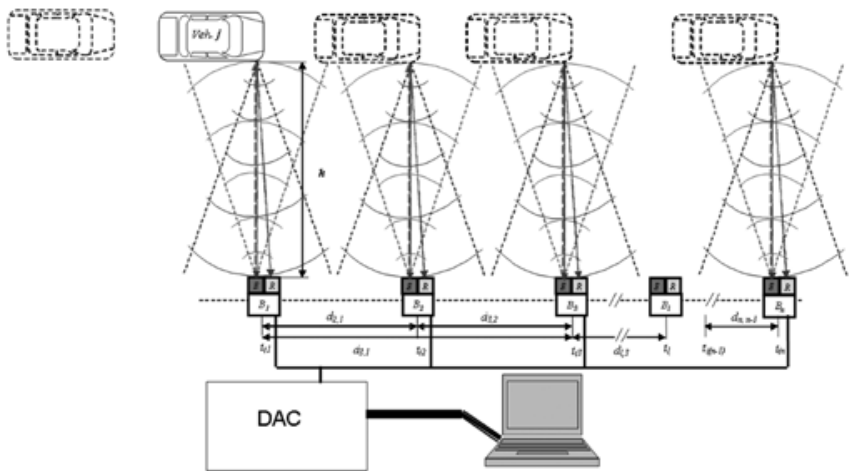

FIG. 2: Esquema de bloques del VUAE 


\section{DESCRIPCIÓN DEL SISTEMA VUAE}

\subsection{Barreras Emisor-Receptor de Ultrasonidos $\left(E_{-}-R_{\text {ult }}\right)$}

El VUAE está compuesto por $n(n \geq 2)$ parejas de $E-R_{u l l^{\prime}}$ situadas en el mismo eje. Cada pareja de $E-R_{u l t}$ se coloca en un trípode con suficiente apoyo para que las vibraciones no provoquen movimientos en él con facilidad. Los emisores de las $n$ parejas $E-R_{u l t}$ generan $n$ barreras de ultrasonidos, se han utilizado emisores y receptores MURATA [3]. El modelo utilizado para los emisores es el M40S4 S. Los receptores captan los ecos cuando el vehículo atraviesa las barreras, se han utilizado MURATA del modelo M40S4 R. El registro de los instantes de tiempo de captación de los ecos recibidos y la distancia entre cada una de las $n$ barreras de ultrasonidos permite la estimación de la velocidad del vehículo con alta exactitud si se afina lo suficiente en la medida del instante de llegada de los ecos a los $E-R_{u l t}$.

La distancia entre cada $E-R_{u l t}$ puede ser variable pero hay que superar un mínimo entre ellos. Esta distancia mínima debe ser tal que un $E-R_{u l t}$ no pueda recibir ecos de una barrera generada por otro $E-R_{u l t}$ adyacente. Esto implica que cuanto más estrechos sean los lóbulos de emisión y recepción de los $E-R_{u l t}$ más cerca se podrán colocar entre sí los $E-R_{u l t}$. Con los emisores y receptores de MURATA M40S4 R/S, que tienen un ángulo de visión (directivity) de $\pm 40^{\circ}$, este ángulo es reducido incorporando un cono $\pm 20^{\circ}$ lo que implica que la distancia

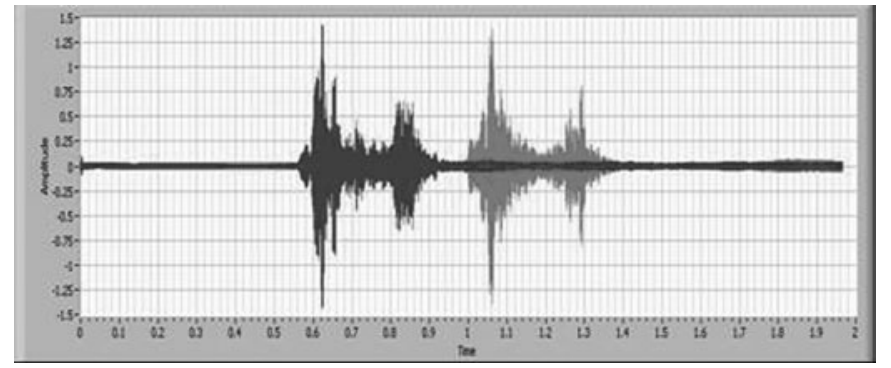

FIG. 3: Señales en el momento de la detección

entre barreras tenga que ser superior a 3,64 m.

Cada barrera $B_{l}$ genera un pulso cuando llega un eco procedente del paso de un vehículo que se usará para sincronizar la adquisición de las señales.

En el emisor M40S4 S, está emitiendo de forma constante una señal a $40 \mathrm{kHz}$. En el receptor la señal captada por el M40S4 $\mathrm{R}$, es amplificada, filtrada, rectificada [2]. Con la ganancia en el acondicionamiento de la señal pueden detectarse vehículos hasta 5-6 $\mathrm{m}$ de distancia del emisor, aunque en el prototipo desarrollado se ha ajustado para que sean detectados vehículos hasta $5 \mathrm{~m}$ de distancia del emisor. Posteriormente se toma la envolvente de la señal y se introduce en un derivador para que se produzca un incremento de señal, únicamente cuando se produzca una alteración en la "vista" del receptor.
TABLA I: M40S4 R/S ESPECIFICACIONES

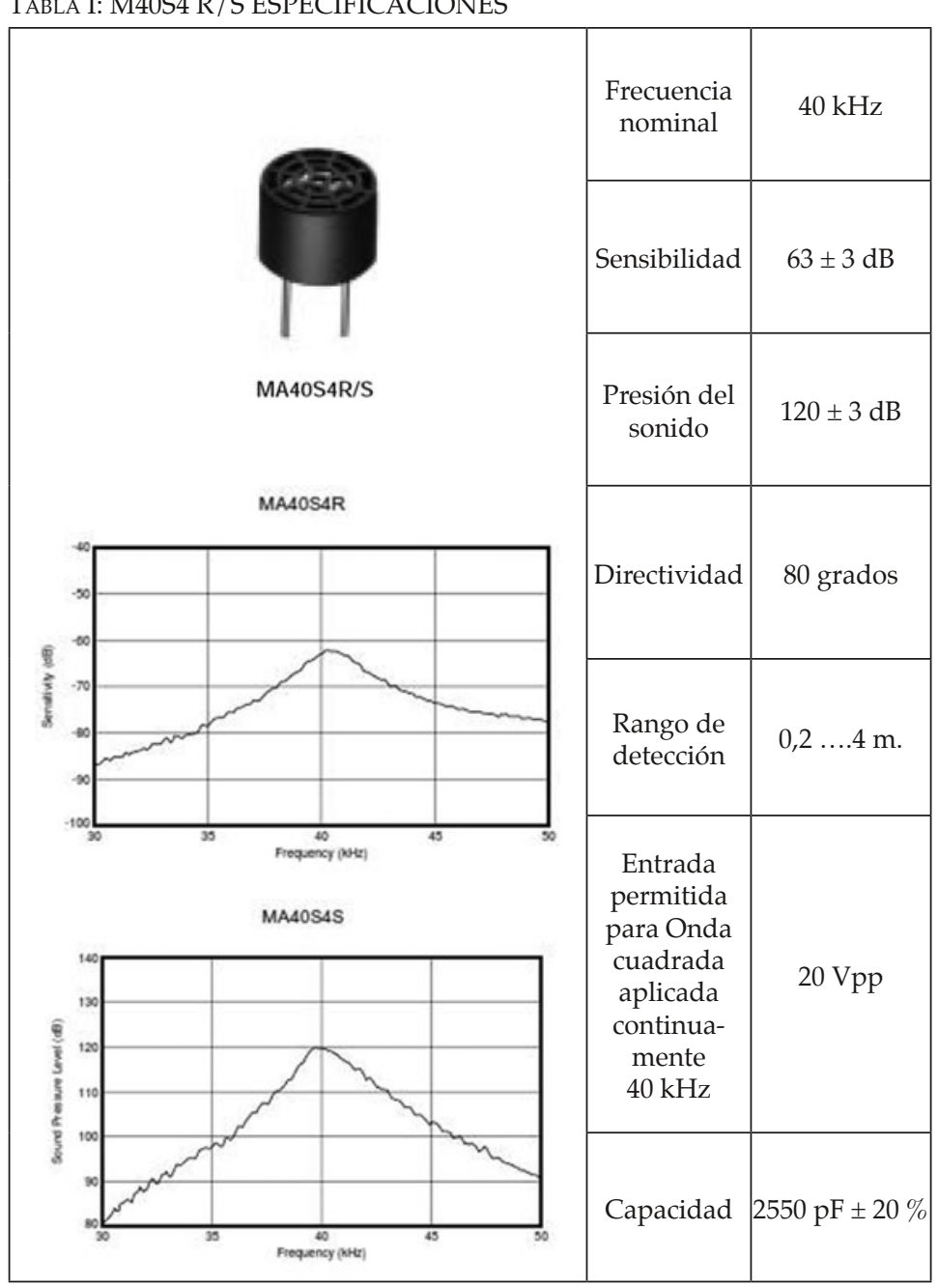

Dicha señal lleva a la saturación a un amplificador con el fin de detectar el vehículo con la máxima rapidez. La salida del amplificador se aplica a un comparador de nivel donde puede ajustarse el nivel de sensibilidad de detección en cada barrera. La señal del comparador se aplica a un multivibrador monoestable para proporcionar un pulso único de $300 \mathrm{~ms}$ cada vez que sea detectado un vehículo. Dicho pulso será usado para sincronizar adecuadamente el sistema de adquisición de datos, DAQ. Dichas señales serán procesadas para calcular la velocidad del vehículo. En la FIG. 3 se muestran las señales en el momento de detección de vehículos obtenidas en una implementación del VUAE con dos barreras. El procesamiento de estas señales permitirá realizar la medida de velocidad del vehículo.

En la Tabla I se resumen las características de los emisores y receptores de ultrasonidos utilizados.

\section{2 Sistema de adquisición de datos (DAQ)}

En el VUAE dispone de un DAQ NI-USB-6210 de National Instruments ${ }^{\mathrm{TM}}$. El $D A Q$ se encarga de las señales de cada barrera y de medir el retardo entre ellas con suficiente exactitud. La sincronización en la adquisición se realiza apoyándose en los contadores del $D A Q$. El $D A Q$ introduce toda la información al computador. Esta medida de tiempo entre ecos requiere un procesado de señal [4] y un tratamiento matemático de las señales adquiridas. En la Tabla II se muestran las características más importantes [5] de los subsistemas utilizados del NI-USB-6210. 
TABLA II: NI-USB-6210

\begin{tabular}{|c|c|}
\hline \multicolumn{2}{|c|}{ CONTADORES } \\
\hline Número de contadores & 2 \\
\hline Resolución & 32 bits \\
\hline Medidas de los contadores & $\begin{array}{c}\text { Cuenta de flancos, pulsos, } \\
\text { semi-periodo, periodo, } \\
\text { separación entre dos flancos }\end{array}$ \\
\hline Salida de los contadores & $\begin{array}{c}\text { Pulso, Tren de pulsos, Divisisor } \\
\text { de frecuencia, Tiempo de } \\
\text { muestreo equivalente }\end{array}$ \\
\hline Relojes Base internos & $\begin{array}{c}80 \mathrm{MHz}, 20 \mathrm{MHz}, 0.1 \mathrm{MHz} \\
50 \mathrm{ppm}\end{array}$ \\
\hline Precisión de los relojes base & ENTRADAS ANALÓGICAS \\
\hline \multicolumn{2}{|c|}{ 16 No diferenciales, 8 } \\
Diferenciales
\end{tabular}

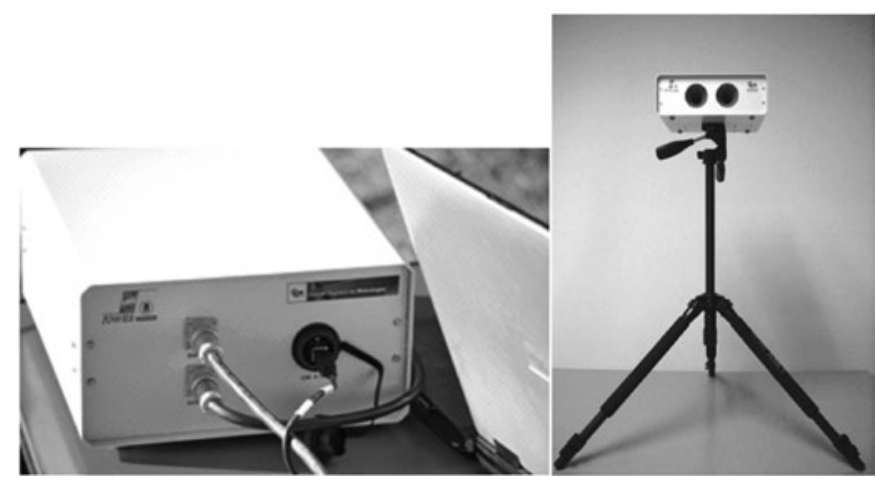

FIG. 4: Equipo para dos barreras y vista de un cabezal ultrasónico que contiene los transductores piezoeléctricos

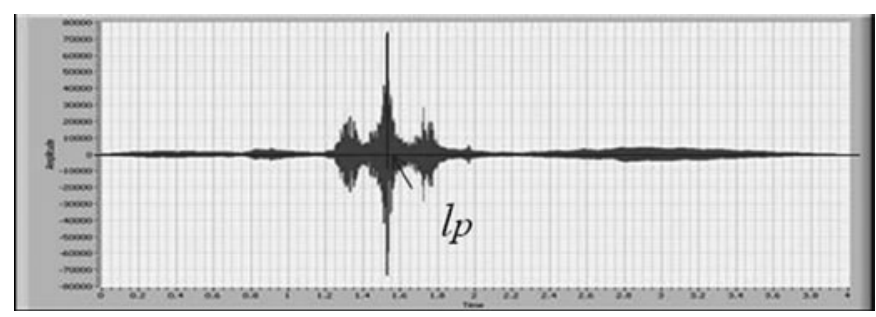

FIG. 5: Correlación cruzada de las señales de dos barreras contiguas
En la FIG. 4 se muestra el equipo electrónico que contiene dos circuitos, uno para cada barrera y un cabezal ultrasónico que es el que creará la barrera ultrasónica.

\subsection{Software}

El software de adquisición de datos y control del VUAE se ha desarrollado íntegramente con el lenguaje de programación LabVIEW тм [6], los datos de entrada al programa son los siguientes:

- Distancia entre barreras (m)

- Tipo de medida (limitadas ó indefinidamente)

- Fichero de almacenamiento de datos de medida

Se describe a continuación la secuencia de acciones en la ejecución del software del $V U A E$, se apoya dicha descripción en la FIG. 2. El operador del VUAE, arranca el programa en el computador y comienzan a recogerse los instantes de recepción de ecos en cada barrera para cada vehículo $j, t_{j k}$ para $k=1, \ldots, n$ (si hay $n$ barreras). Los instantes de detección de los ecos se calculan por medio de la correlación cruzada. En la FIG. 5 se muestra la correlación cruzada de las señales adquiridas en la FIG. 3.

El VUAE obtendrá una cantidad $N$ de medidas de velocidad. Cuando un vehículo cualquiera, $V_{\text {eh.j. }}$ intercepta cada una de las $n$ barreras de ultrasonidos, se detectan los ecos producidos en cada receptor del $E-R_{u l t}$ y se registran por medio del $D A Q$ los instantes de detección del eco $t_{j k}$ para $k=1$, $2 \ldots, n$.

Por otra parte se dispone de los datos de las distancias entre las diferentes barreras de ultrasonidos $d_{k, l}$ para $k=2,3, \ldots$, $n$ y $l=1,2, \ldots, n-1$.

Se adquieren y se filtran las señales de los cabezales, por ejemplo: $\mathrm{B}_{i^{\prime}}$ señal $\mathrm{x}(\mathrm{k})$ y $\mathrm{B}_{\mathrm{i}+1^{\prime}}$ señal $\mathrm{y}(\mathrm{k})$. Estas señales tienen $\mathrm{N}$ muestras muestreadas a una frecuencia de muestreo fs $(\mathrm{Hz})$

Se realiza la autocorrelación entre $x(k)$ e $y(k)$ y se calcula el retardo entre señales y la velocidad según las expresiones de Ec. 3.

$\left.\begin{array}{l}r_{x y}(l)=\sum_{k=0}^{N} x(k) \cdot y(k-l) l=0,1,2, \ldots, N \\ t_{i+1}-t_{i}=\left(N-l_{p}\right) \cdot \frac{1}{f_{s}}\end{array}\right\} v=\frac{d_{b}}{t_{i+1}-t_{i}}$

Ec. 3

Se calcula el estimador de velocidad para el $V_{\text {eh.j }}$ según la Ec. 4:

$\hat{v}_{j}=\frac{\sum_{k=2}^{n}\left(\sum_{l=1}^{k-1} \frac{d_{k, l}}{t_{j k}-t_{j l}}\right)}{\frac{n}{2}(n-1)}$

Ec. 4 
- Se registra la estimación de velocidad $\hat{v}_{j}$ y los instantes $t_{j k}$.

- Puede darse la opción de tomar un número de medidas determinadas ó tomar medidas indefinidamente hasta que el operador determine finalizar la recogida de medidas.

- Cuando se llegue al número de medidas determinado ó cuando el operador ordene parar la recogida, el $V U A E$ dejaría de tomar medidas.

En la FIG. 6 se muestra el panel frontal del software de medida.

\subsection{Estimación de la exactitud del sistema}

Es muy importante estimar el error máximo que se puede tener en la medida de la velocidad. Este error depende fundamentalmente de:

- $\quad$ Error en la medida de la distancia entre barreras

- La falta de paralelismo entre las barreras

- La diferente forma de onda entre las señales de cada barrera barreras

- El instante de muestreo de la señal

Se refleja en la Tabla III el error estimado, en el caso peor, para diferentes velocidades suponiendo que el vehículo pasa a una distancia $h=5 m$ del $E-R_{u l t}$. Se suponen sólo dos barreras cuya distancia entre ellas $\mathrm{d}_{2,1}=8 \mathrm{~m}$. La variación de la distancia prefijada entre las barreras, $\Delta \mathrm{d}_{\mathrm{b}^{\prime}}$ introduce una variación en la medida de tiempo $\Delta t$, que provoca un error en la medida de velocidad. Se muestra en la Tabla III la estimación del error realizada.

La falta de paralelismo introduce un $\Delta \mathrm{d}_{\mathrm{b}^{\prime}}$ variación en la distancia entre las barreras, introduce una variación en la medida de tiempo $\Delta t$, que provoca un error en la medida de velocidad. Se ha dotado al VUAE de un sistema laser para colocar las barreras paralelas entre sí consiguiendo un error

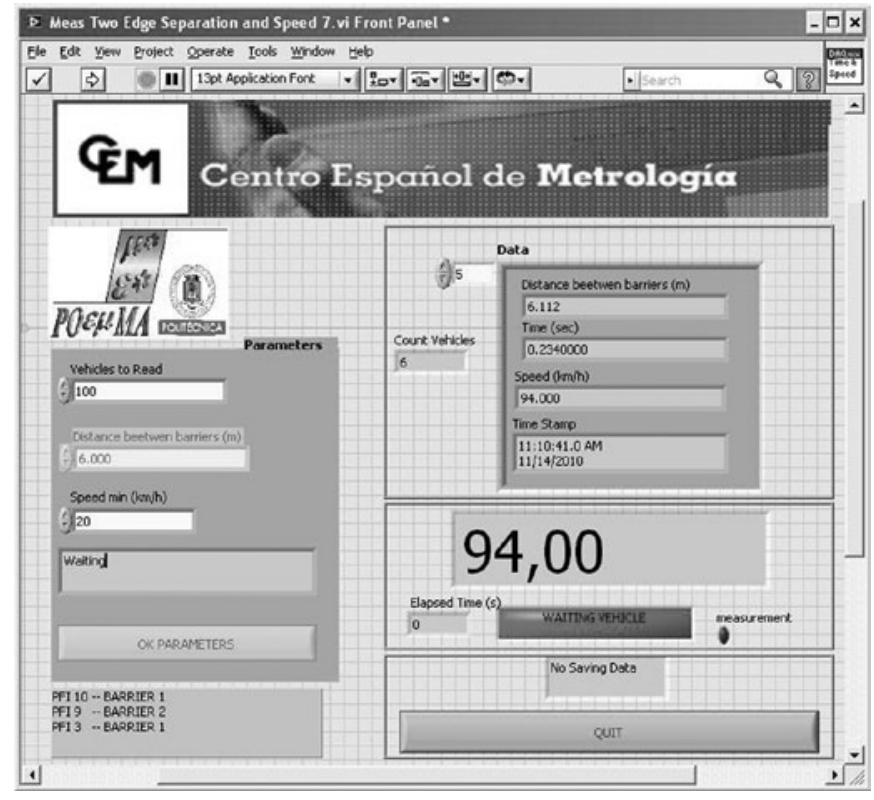

FIG. 6: Panel frontal del software

máximo de anti-paralelismo de $\pm 1^{\circ}$ Se muestra en la Tabla III la estimación del error realizada.

Se estima que la diferencia en la forma de onda entre las señales captadas en cada una de las barreras puede desplazar el máximo de la autocorrelación en $100 \mu$ s como máximo. Se muestra en la Tabla III la estimación del error realizada.

Existe una fuente de error en la incertidumbre en el instante de muestreo de la señal. Este error se refiere al hecho de que el instante de muestreo en el que se ha tomado el máximo de la real no coincida con el máximo real de la señal. Se estima que el máximo de las señales de las barreras puedan estar desviadas hasta 5 muestras. Esto haría que se llegase a desplazar hasta 10 muestras el máximo de la auto-correlación. Se muestra en la Tabla III la estimación del error realizada.

\begin{tabular}{|c|c|c|c|c|c|}
\hline $\begin{array}{c}\text { Velocidad del } \\
\text { Vehiculo }(\mathrm{km} / \mathrm{h})\end{array}$ & $\begin{array}{c}\text { Error de Distancia entre } \\
\text { Barreras }(\mathrm{km} / \mathrm{h})\end{array}$ & $\begin{array}{c}\text { Error de Falta de } \\
\text { Paralelismo entre } \\
\text { Barreras }(\mathrm{km} / \mathrm{h})\end{array}$ & $\begin{array}{c}\text { Error de Forma en las } \\
\text { Señales }(\mathrm{km} / \mathrm{h})\end{array}$ & $\begin{array}{c}\text { Error en el instante de } \\
\text { muestreo }(\mathrm{km} / \mathrm{h})\end{array}$ & $\begin{array}{c}\text { Sumatorio de } \\
\text { Errores }(\mathrm{km} / \mathrm{h})\end{array}$ \\
\hline 25 & 0,00 & 0,01 & 0,00 & 0,00 & 0,01 \\
\hline 50 & 0,00 & 0,02 & 0,01 & 0,00 & 0,03 \\
\hline 75 & 0,00 & 0,03 & 0,02 & 0,00 & 0,06 \\
\hline 100 & 0,00 & 0,04 & 0,03 & 0,001 & 0,09 \\
\hline 125 & 0,01 & 0,05 & 0,05 & 0,01 & 0,12 \\
\hline 150 & 0,01 & 0,06 & 0,08 & 0,01 & 0,16 \\
\hline 175 & 0,01 & 0,07 & 0,11 & 0,01 & 0,20 \\
\hline 200 & 0,01 & 0,08 & 0,14 & 0,02 & 0,25 \\
\hline 225 & 0,01 & 0,09 & 0,18 & 0,02 & 0,30 \\
\hline 250 & 0,01 & 0,11 & 0,22 & 0,03 & 0,36 \\
\hline 275 & 0,01 & 0,12 & 0,31 & 0,03 & 0,42 \\
\hline 300 & 0,01 & 0,13 & 0,37 & 0,04 & 0,48 \\
\hline 325 & 0,02 & 0,14 & 0,42 & 0,04 & 0,56 \\
\hline 350 & 0,02 & 0,15 & 0,49 & 0,05 & 0,63 \\
\hline 375 & 0,02 & 0,16 & 0,55 & 0,06 & 0,71 \\
\hline 400 & 0,02 & 0,17 & 0,80 & \\
\hline
\end{tabular}


En la FIG. 7 se muestra una gráfica del error estimado donde se puede ver el impacto de cada una de las fuentes de error consideradas.

\section{VERIFICACIÓN DEL FUNCIONAMIENTO DEL CCVUAE}

Hemos realizado medidas con el VUAE en una pista de pruebas. Dichas medidas se han contrastado con un sistema de referencia basado en dos cables piezoeléctricos situados en paralelo a una distancia predefinida y captando los instantes en las ruedas del vehículo impactan con cada uno de los cables. El error máximo estimado del sistema de medida de cables es del $0,1 \%$. Se calcula la velocidad según la Ec. 1. En la FIG. 8 se muestra la estructura interna de los cables piezoeléctricos [7] y en el material piezoeléctrico sigue la ecuación matricial Ec. 5 de la densidad de carga:

$\left[\begin{array}{l}D_{x} \\ D_{y} \\ D_{z}\end{array}\right]=\left[\begin{array}{llllll}d_{11} & d_{12} & d_{13} & d_{14} & d_{15} & d_{16} \\ d_{21} & d_{22} & d_{23} & d_{24} & d_{25} & d_{26} \\ d_{31} & d_{32} & d_{33} & d_{34} & d_{35} & d_{36}\end{array}\right]\left[\begin{array}{c}\sigma_{x x} \\ \sigma_{y y} \\ \sigma_{z z} \\ \tau_{y z} \\ \tau_{z x} \\ \tau_{x y}\end{array}\right]$
Particularizando para el cable, la densidad de carga generada en condiciones próximas a cortocircuito será: $\mathrm{D}=$ $\mathrm{d} 3 \mathrm{n} \cdot \mathrm{Xn}$, con $X_{n}$ la presión $\left(\mathrm{N} / \mathrm{m}^{2}\right)$ en el eje $n$.

La tensión en circuito abierto será: $V=g_{3 n} \cdot X_{n} t$, siendo $t$ el espesor del material piezoeléctrico.

En la FIG. 9 se muestra un ejemplo de la respuesta del cable enterrado al paso de un vehículo de 5 ejes donde el primer pico es el primer eje, seguido de dos ejes del camión más los dos últimos ejes.

En una instalación real, los cables piezoeléctricos estarán enterrados bajo el pavimento en las condiciones especificadas por el fabricante [8].

Los resultados de la comparación entre las medidas del sistema de referencia y las medidas de velocidad tomadas con VUAE se muestran en la Tabla IV y. El error mostrado es la suma del error del VUAE y el error del sistema de cable piezoeléctrico. Para confeccionar dicha tabla se han realizado 32 medidas al paso de 32 vehículos.

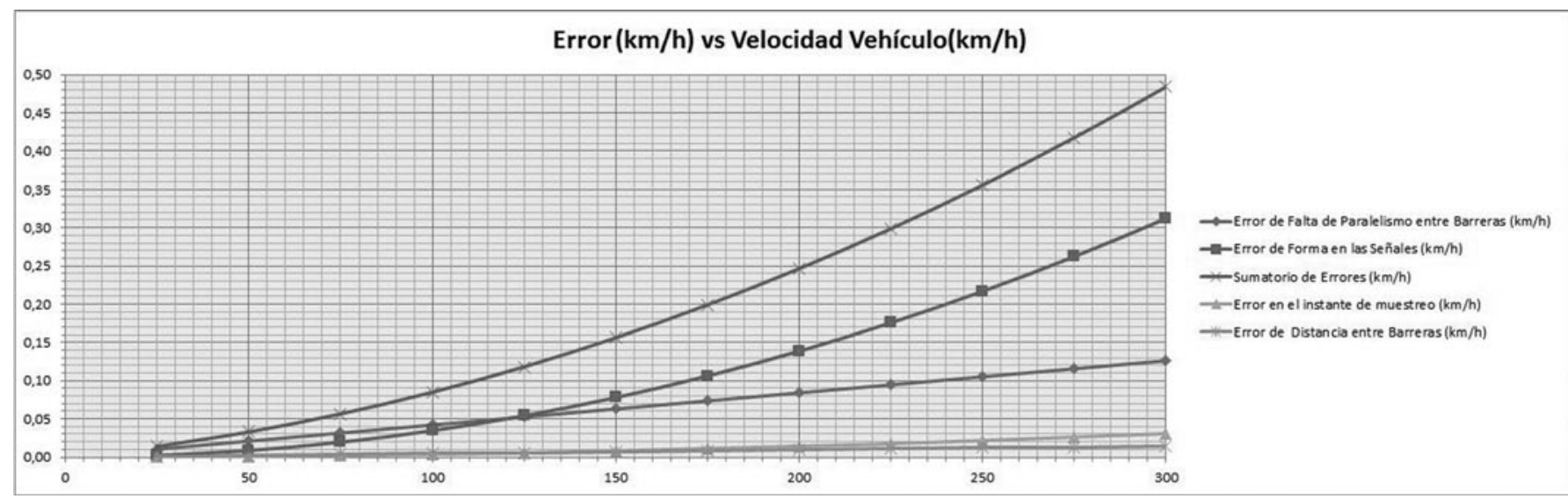

FIG. 7 Errores estimados y su impacto sobre el sumatorio de errores

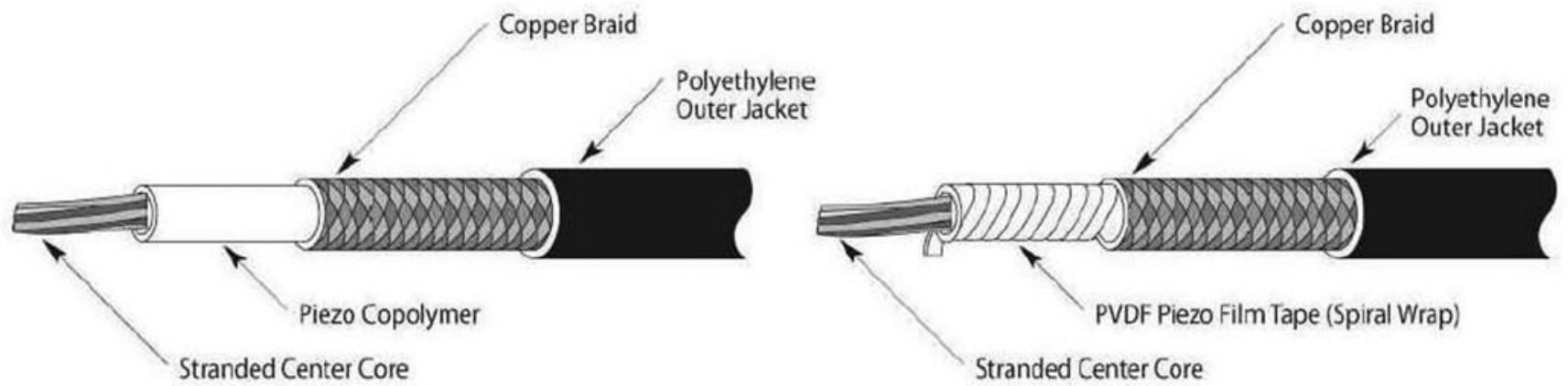

20AWG Cable-Copolymer

20AWG Cable-Spiral Wrap

FIG. 8: Estructura interna de los cables piezoeléctricos P/N: 1005801-1 de Measurement Specialties, Inc. 


\section{CONCLUSIONES}

- Se ha conseguido un sistema basado en dispositivos piezoeléctricos que proporciona unas especificaciones técnicas de alto nivel para la medida de velocidad de vehículos.

- $\quad$ Se deduce que con la concepción del VUAE se evitan algunos de los problemas descritos anteriormente en el estado de la técnica y además supera con creces los requerimientos iniciales expuestos para su diseño.

- Ya no es necesaria una alineación previa entre el emisor y el receptor para poder interceptar el paso del vehículo, evitando el gasto excesivo de tiempo en el ajuste de la alineación antes de comenzar el proceso de medida. Sólo hay que poner las barreras paralelas entre sí, y con el sistema láser de alineamiento se realiza en muy poco tiempo en cualquier punto kilométrico.

- $\quad$ El consumo de corriente de los $E-R_{u l t}$ y del resto del equipamiento es bajo, por lo que se podría alimentarse todo el sistema desde la alimentación del computador. Para $n=2$ barreras se ha medido un consumo de 150 $\mathrm{mA}$ aproximadamente.

- Las vibraciones de un vehículo pesado no afectan al $E-R_{u l t}$ porque no existe la alineación entre emisor y receptor. No es necesario fijar los $E-R_{u l t}$ al suelo por lo que se evitan las instalaciones permanentes del sistema. Se minimiza el problema de la desalineación.

- No se necesita vehículo de referencia para tomar medidas de velocidad.

- $\quad$ Reduce considerablemente el índice de peligrosidad porque el/los operario/s no tienen que cruzar la vía para colocar el equipamiento.
Tabla IV: Error estimado entre Cables Piezoeléctricos y VUAE

\begin{tabular}{|c|c|c|c|}
\hline Velocidad Cables $(\mathrm{km} / \mathrm{h})$ & Velocidad VUAE $(\mathrm{km} / \mathrm{h})$ & Diferencia $(\mathrm{km} / \mathrm{h})$ & Error Max.(km/h) \\
\hline 37,17 & 37,28 & $-0,11$ & 0,5 \\
\hline 57,06 & 56,79 & 0,27 & \\
\hline 89,69 & 89,95 & $-0,26$ & \\
\hline 70,62 & 70,63 & $-0,01$ & \\
\hline 87,78 & 87,7 & 0,08 & \\
\hline 69,74 & 69,24 & 0,5 & \\
\hline 87,22 & 87,22 & 0 & \\
\hline 61,95 & 61,43 & 0,52 & \\
\hline 60,75 & 60,83 & $-0,08$ & \\
\hline 59,52 & 59,13 & 0,39 & \\
\hline 57,25 & 57,2 & 0,05 & \\
\hline 52,36 & 51,84 & 0,52 & \\
\hline 80,22 & 79,93 & 0,29 & \\
\hline 50,29 & 50,2 & 0,09 & \\
\hline 85,18 & 85,1 & 0,08 & \\
\hline 51,84 & 51,34 & 0,5 & \\
\hline 79,72 & 79,93 & $-0,21$ & \\
\hline 42,98 & 42,96 & 0,02 & \\
\hline 81,04 & 81,37 & $-0,33$ & \\
\hline 43,83 & 43,85 & $-0,02$ & \\
\hline 71,09 & 71,27 & $-0,18$ & \\
\hline 41,85 & 41,6 & 0,25 & \\
\hline 57,88 & 58,25 & $-0,37$ & \\
\hline 70,34 & 70,16 & 0,18 & \\
\hline 63,77 & 64,04 & $-0,27$ & \\
\hline 63,22 & 63,15 & 0,07 & \\
\hline 60,59 & 60,48 & 0,11 & \\
\hline 52,68 & 53,06 & $-0,38$ & \\
\hline 53,54 & 53,42 & 0,12 & \\
\hline 45,84 & 45,82 & 0,02 & \\
\hline 48,08 & 48,57 & $-0,49$ & \\
\hline 46,02 & 46,09 & $-0,07$ & \\
\hline
\end{tabular}

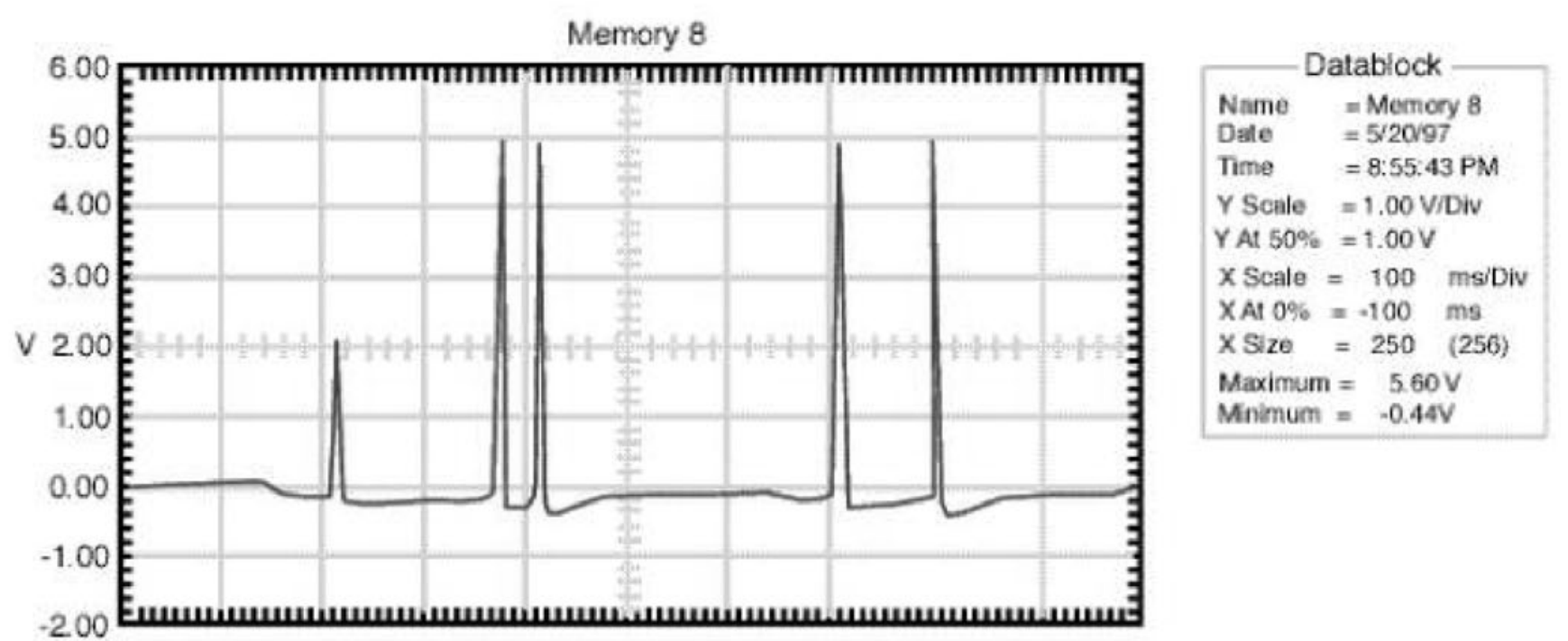

$-100 \mathrm{~ms}$

$100 \mathrm{~ms} / \mathrm{Div}$

FIG. 9: Señal camión de 5 ejes del cable P/N: 1005801-1 de Measurement Specialties, Inc. 


\section{REFERENCIAS}

1. Muñoz, R.; Reale, C.; Caniglia, C. Desarrollo de un cinemómetro de referencia con tecnología Láser INTI (2004)

2. Ultrasonic Sensor Application Manual. MURATA LTD (2008)

3. Piezoelectric Ceramics (PIEZOTITE®) Sensors MURATA LTD (2008)

4. F.J. Jiménez, J. De Frutos; Virtual instrument for measurement, processing data, and visualization of vibration patterns of piezoelectric devices. Elsevier: Computer Standards \& Interfaces 27 (2005) 653-663

5. NI $621 x$ Specifications. National Instruments Corporation (2008)

6. http://www.ni.com/labview/

7. Piezo Film Sensors Technical Manual. Measurement Specialties, Inc. (2006)

8. Lahtinen, R; Muukkonen, $\mathrm{T}$; Koskinen A piezopaint-based sensor for monitoring structure dynamics, J. Smart Materials \& Structures 16(6) 25712576 (2007)

9. Li ZX, Yang XM, Li ZJ. Application of cement-based piezoelectric sensors for monitoring traffic flows. J. Transp. Eng ASCE 132 (7) 565-573 (2006)

10. J D Turner and L Austin. Sensors for automotive telematics; Meas. Sci. Technol. 11 (2000) R58-R79

11. Iaquinta, J; Merliot, E; Cottineau, LM. Piezoelectric sensors for weigh-inmotion systems: J. Testing And Evaluation 32 (6): 476-483 (2004)

Recibido: $14 / 07 / 2011$

Aceptado: 22/11/2011 